\title{
Enhancing Fourier Volume Rendering Using Contour Extraction
}

\author{
Zoltán Nagy, Marcin Novotni, and Reinhard Klein \\ Department for Computer Science II, Bonn University, 53117 Bonn, Germany \\ \{zoltan, marcin,rk\}@cs.uni-bonn.de \\ http://cg.cs.uni-bonn.de
}

\begin{abstract}
Fourier Volume Rendering (FVR) has received considerable attention in volume visualization during the last decade due its $O\left(N^{2} \log N\right)$ rendering time complexity, where $O\left(N^{3}\right)$ is the volume size. Nevertheless, FVR currently suffers from some quality limiting its usefulness in particular medical applications. The main reason for this is the lack of weighting sample points in dependence of the samples along the integration path. In this work we propose a solution for a special class of problems, namely the extraction and emphasis of contours in volumetric datasets. The accuracy of the illumination of the extracted contours can be derived in an exact manner. Main applications of our method include contour extraction and enhancement of features, noise removal and revealing of important spatial relationships between interior and exterior structures, making it an attractive tool for improved $\mathrm{X}$-ray-like investigations of the given dataset.
\end{abstract}

\section{Introduction}

Volume rendering is a technique for visualizing sampled scalar or vectorial volumes of three dimensions by propagation of light in a participating medium. Two main approaches have established in this area: (i) object space and (ii) Fourier space algorithms. The first class of algorithms was originally introduced by Kajiya [6] in 1984. Since then, this model was steadily improved in both speed and quality using global illumination, graphics hardware, pre-integration strategies and Wavelet compression (see e.g. [12, 4] and [7]). Since working in object space, these algorithms allow for modelling light transport in a variety of ways, involving transfer functions and physical parameters like albedo, interreflection, material density, gradients, etc. This class of volume rendering models has in general a rendering time complexity of $O\left(N^{3}\right)$, where $N^{3}$ is the volume size, since the whole dataset has to be sampled entirely per frame.

The second class of algorithms work in Fourier space. Introduced by Levoy [8] and Malzbender 9], this method sacrifices some features -which object space algorithms offer- against rendering speed. In FVR, the original dataset is 3D Fourier transformed in $O\left(N^{3} \log N\right)$ time in the preprocessing phase. During rendering, a plane through the center of the Fourier transformed data representation is sampled perpendicularly to the viewing direction and 2D inverse Fourier transformed in $O\left(N^{2} \log N\right)$ time. The Fourier Projection-Slice Theorem tells us, that 
the so obtained image contains an unweighted projection of the sample points of the input dataset from the given view. This leads to occlusion free X-ray like images, since ray attenuation can not be modelled in dependence of the actively cumulated opacity of the voxels. It was soon recognized, that occlusion-free projections are difficult to interpret and a X-ray like linear depth cueing illumination model with directional shading was introduced [8]. The latter method can support the observer in estimating positions of anatomical entities, but projections remain occlusion free.

In this work we eliminate some disadvantages of standard FVR methods. In particular, we extract material boundaries on surfaces in Fourier space. This problem was already considered for medical questions in object space [12], rather than Fourier space. In this work, we show, how this technique can be derived and implemented in Fourier space. We show three important applications which makes our technique attractive: (i) extraction of object, rather than screen-space contours, (ii) noise reduction and (iii) removal of non-contributing samples to obtain better visibility of the contours. To accomplish these we adopt the reflection equation for shading in context of FVR [3] formulated in terms of Spherical Harmonics. The main difference is the novel interpretation of the terms appearing in the equation: (i) instead of the incoming light used originally to shade the surface the gradient magnitude is plugged in to emphasize areas of high contrast and (ii) instead of the cosine projection term we use a sine transfer function to highlight surfaces with normals perpendicular to the viewing direction (cf. Section 3 for details). Our technique is of special interest for radiologists who work with X-ray like projections of the given dataset and who want to obtain a better insight to spacial relationships of the investigated medical material.

\section{Shading Using Spherical Harmonics}

Spherical harmonic (SH) approximation of an illumination model allows for shading of the dataset without recalculating the entire 3D transform per frame. The method derived here is a generalized view on the methods of Entezari et al. [3] and Ramamoorthi and Hanrahan [11. Their derivation is tailored to lighting voxels/surfaces; we, however, deduce a method for contour extraction.

Normalized SHs are a rotational invariant group of functions that form an orthonormal basis on the unit sphere. While mostly described in complex form, a real representation is available saving memory representing the imaginary part [3]. Any function of finite energy on the sphere may be approximated to any degree of accuracy in terms of SHs $Y_{l m}(\theta, \phi)$ using the expansion

$$
f(\theta, \phi)=\sum_{l} \sum_{m} f_{l m} Y_{l m}(\theta, \phi)
$$

where $f_{l m}=\int_{0}^{2 \pi} \int_{0}^{\pi} f(\theta, \phi) Y_{l m}^{*}(\theta, \phi) \sin \theta d \theta d \phi$. The radiance on a surface (or more specifically, on a voxel position) in its original setting is given by

$$
E=\int_{\Omega_{i}^{\prime}} L\left(\omega_{i}\right) \rho\left(\omega_{i}^{\prime}, \omega_{o}^{\prime}\right) \max \left(\cos \theta_{i}^{\prime}, 0\right) d \omega_{i}^{\prime}
$$


where subscript $i(o)$ denotes the incoming (outgoing) light direction, the global (local) coordinate system is unprimed (primed), $L\left(\omega_{i}\right)$ is the incoming light, $\rho\left(\omega_{i}^{\prime}, \omega_{o}^{\prime}\right)$ is the BRDF (bidirectional reflection distribution function) over the upper hemisphere $\Omega_{i}^{\prime}$ and $\max \left(\cos \theta_{i}^{\prime}, 0\right)$ is a transfer function preventing the surface from being lit from behind. For the case that diffuse shading is assumed ( $\rho=1 / \pi=$ const. $)$, and an arbitrary transfer function $f(\theta, \phi)$ is chosen, we obtain omitting $\rho$

$$
E=\int_{\Omega_{i}^{\prime}} L\left(\omega_{i}\right) f\left(\omega_{i}^{\prime}\right) d \omega_{i}^{\prime}
$$

By expanding $L\left(\omega_{i}\right)=\sum_{l} \sum_{m} L_{l m} Y_{l m}\left(\omega_{i}\right)$ and $f\left(\omega_{i}^{\prime}\right)=\sum_{l} \sum_{m} f_{l m} Y_{l m}\left(\omega_{i}^{\prime}\right)$, substitution into equation (3) leads to the general formulation

$$
E=\sum_{l} \sum_{m} \sum_{p} \sum_{q} L_{l m} f_{p q} \int_{\Omega_{i}^{\prime}} Y_{l m}\left(\omega_{i}\right) Y_{p q}\left(\omega_{i}^{\prime}\right) d \omega_{i}^{\prime}
$$

Note, that two different coordinate systems are assumed here (primed and unprimed). To rotate the primed system into the unprimed one, the canonical rotation formula using the coefficients related to the matrix of the rotation group $S O(3) 11$

$$
Y_{l m}\left(\omega_{i}\right)=Y_{l m}\left(R_{\alpha \beta}\left(\omega_{i}^{\prime}\right)\right)=\sum_{m^{\prime}=-l}^{l} D_{m m^{\prime}}^{l}(\alpha) e^{i m \beta} Y_{l m^{\prime}}\left(\omega_{i}^{\prime}\right)
$$

is used, where $R_{\alpha \beta}\left(\omega_{i}^{\prime}\right)$ represents the rotation into the unprimed coordinate system. Applying this rotation in equation (4) leads to

$$
E=\sum_{l} \sum_{m} \sum_{p} \sum_{q} L_{l m} f_{p q} \sum_{m^{\prime}} D_{m m^{\prime}}^{l}(\alpha) e^{i m \beta} \int_{\Omega_{i}^{\prime}} Y_{l m^{\prime}}\left(\omega_{i}^{\prime}\right) Y_{p q}\left(\omega_{i}^{\prime}\right) d \omega_{i}^{\prime}
$$

Due to the orthogonality relationship of $\mathrm{SH}$ the integral portion is nonzero iff $l=p$ and $m^{\prime}=q$. Using the relations $D_{m 0}^{l}(\alpha) e^{i m \beta}=\sqrt{\frac{4 \pi}{2 l+1}} Y_{l m}(\alpha, \beta)$ and $L_{l m}=Y_{l m}\left(\theta_{L}, \phi_{L}\right)$ 3] we obtain a specialized formulation for functions $f$ with no asimuthal dependence $\left(f_{p q}\right.$ is zero for $\left.q \neq 0\right)$ :

$$
E=\sum_{l, m, p} L_{l m} f_{p 0} \sum_{m^{\prime}} D_{m m^{\prime}}^{l}(\alpha) e^{i m \beta} \delta_{l, p} \delta_{m^{\prime}, 0}=\sum_{l, m} \sqrt{\frac{4 \pi}{2 l+1}} Y_{l m}\left(\omega_{L}\right) f_{l 0} Y_{l m}(\alpha, \beta)
$$

To use latter equation for contour extraction, three requirements have to be met. First, appropriate SH coefficients $f_{l 0}$ have to be found which model the brightness of a contour in dependence of the viewing vector and the local gradient. An explicit formula is derived for this in the appendix. Second, note that equation (7) is derived for diffuse shading, i.e. the illumination is independent of the viewer. Fortunately, for contour extraction, we can set the light direction $\omega_{L}$ collinear to 
the viewing direction $\omega_{V}: \omega_{V}=\omega_{L}$, therefore this is implicitly solved. Thirdly, we want to weight a voxel individually, depending on the gradient magnitude, to remove homogeneous regions and to obtain a high degree of visibility of the contours. This is done in the following section.

\section{Contour Extraction}

Equation (7) is the key equation for general diffuse lighting of a surface, when the lighting distribution and the transfer function can be represented in terms of SH coefficients $L_{l m}$ and $f_{l m}$, respectively. From this general setting, we can derive a special case, which allows for the enhancement of contours in Fourier space. We regard a sample in the volume as a contour sample, iff $\left\langle\omega_{V}, \omega_{N}\right\rangle=0$, i.e. the viewing vector $\omega_{V}$ is perpendicular to the normal $\omega_{N}$ of the given sample. In the following, a metric of the degree of how much the active sample is likely to be a contour sample is given by the function $f$ :

$$
f\left(\theta_{i}^{\prime}, \phi_{i}^{\prime}\right)=f\left(\theta_{i}^{\prime}\right)=\sin \left(\theta_{i}^{\prime}\right)^{2 k}, k \in \mathbf{N}_{0}, \theta_{i}^{\prime}=\cos ^{-1}\left(<\omega_{V}, \omega_{N}>\right)
$$

In our experiments, we used $k=8$. The motivation for using this function is to emphasize samples with gradients near-to-orthogonal, in order to induce enough illumination energy for the final projection.

The last thing missing in our considerations is the weighting of the scalar voxel values in dependence of the gradient length. We use a squared version of the gradient length to overemphasize positions with high gradient length:

$$
E_{\text {weighted }}=E \cdot\left\|\overrightarrow{\omega_{N}}\right\|_{2}^{2}=\sum_{l} \sum_{m} \sqrt{\frac{4 \pi}{2 l+1}}\left\|\overrightarrow{\omega_{N}}\right\|_{2}^{2} Y_{l m}\left(\omega_{V}\right) f_{l 0} Y_{l m}\left(\omega_{N}\right)
$$

where $\left\|\overrightarrow{\omega_{N}}\right\|_{2}$ denotes the length of the respective vector $\mathrm{N}$ at the surface (or voxel) position.

\section{Algorithm}

Equation (9) describes the shading at a voxel position in dependence of the local normal $\mathrm{N}$ and the global viewing vector $\mathrm{V}$. Applying the Fourier transform operator $\mathcal{F}\{\}$ results in

$$
\mathcal{F}\left\{E_{\text {weighted }}\right\}=\sum_{l} \sum_{m} \sqrt{\frac{4 \pi}{2 l+1}} Y_{l m}\left(\omega_{V}\right) f_{l 0} \mathcal{F}\left\{\left\|\overrightarrow{\omega_{N}}\right\|_{2}^{2} Y_{l m}\left(\omega_{N}\right)\right\}
$$

The usage of this equation is as follows. During preprocessing, volumes $V_{l m}$ are initialized with their respective voxel values $V_{l m}(x, y, z)=$ $\left\|\overrightarrow{\omega_{N}}\right\|_{2}^{2} Y_{l m}\left(\theta_{x y z}, \phi_{x y z}\right), 0 \leq l \leq M,-l \leq m \leq l$. The 3D Fourier transform is then applied on every volume $\bar{V}_{l m}$. During rendering, we sample slices $S_{l m}\left(x^{\prime}, y^{\prime}\right)$ through the origins of every transformed dataset $\mathcal{F}\left\{V_{l m}\right\}$ perpendicularly to the 
viewing direction $\omega_{V}$. Slice $S_{l m}\left(x^{\prime}, y^{\prime}\right)$ is weighted by $\sqrt{\frac{4 \pi}{2 l+1}} Y_{l m}\left(\omega_{V}\right)$ at every sample point. Finally, the weighted slices are summed up componentwise and the inverse 2D Fourier transform is executed on the result, leading to the desired projection for the current viewing direction.

\section{Results and Discussion}

In figure 1 we compare rendering results of the conventional FVR algorithm $\left(1^{\text {st }}\right.$ and $3^{\text {rd }}$ row) with our method ( $2^{\text {nd }}$ and $4^{\text {th }}$ row), using the head, skull (both obtainable from www . volren.org) and the Visible Female dataset. The examples show applications in three main areas.

Hidden feature emphasis. The head dataset (left column) exemplifies how our algorithm is capable of recovering features when the material density of the input dataset is high. We are able to emphasize transitions between air and skull, skull and cerebrospinal fluid, and boundaries around the corpus callosum.

Noise reduction. The skull (middle column) is an example for an extremely noisy dataset. The projection quality is worsened by the fact, that this property is amplified by "ghosting" artifacts of ordinary FVR. Since noise in more likely contained with low gradient portion, our algorithm removes it significantly. Furthermore, the contours of the teeth appear sharper here than with the traditional method.

Boundary and contrast enhancement. Finally, we were able to recognize some important spatial relationships on the Visible Female dataset (right column) using our method. While ordinary FVR leads to strongly diffuse projections of the dataset, we were able to relate exterior structures, like the run of the skin, the ears, the lips, with interior structures like the (upper and lower) jaw and the spinal court using our algorithm.

All three datasets used have a size of $128^{3}$ voxels, where the second and third one were downsampled to this size in order to reduce memory requirements. Rendering times are identical for every view and dataset, with about $3 \mathrm{fps}$ on an $3.06 \mathrm{GHz}$ Intel P IV with 1 GByte RAM.

A little caveat of our method is the somewhat high memory consumption. When using three non-negative SH coefficients, 15 datasets have to be used with 4 Bytes at each voxel for the real and the imaginary part, respectively. Since we work with real SHs, the input volumes are real, and according to Fourier theory $F(u, v)=F^{*}(-u,-v)$, i.e. the Fourier transformed dataset is symmetric with respect to the origin up to conjugation. Thus, memory can be saved by a factor of two. Lossy methods for further memory reduction like vector compression can be incorporated- this is part of future work.

\section{Conclusion and Future Work}

The intention of this work was to make FVR a more viable alternative in volume visualization, when it comes to explore medical datasets via X-ray like volume rendering techniques. Due to its reduced runtime complexity, the importance of our method in a quantitative sense will grow, when datasets will become larger. 

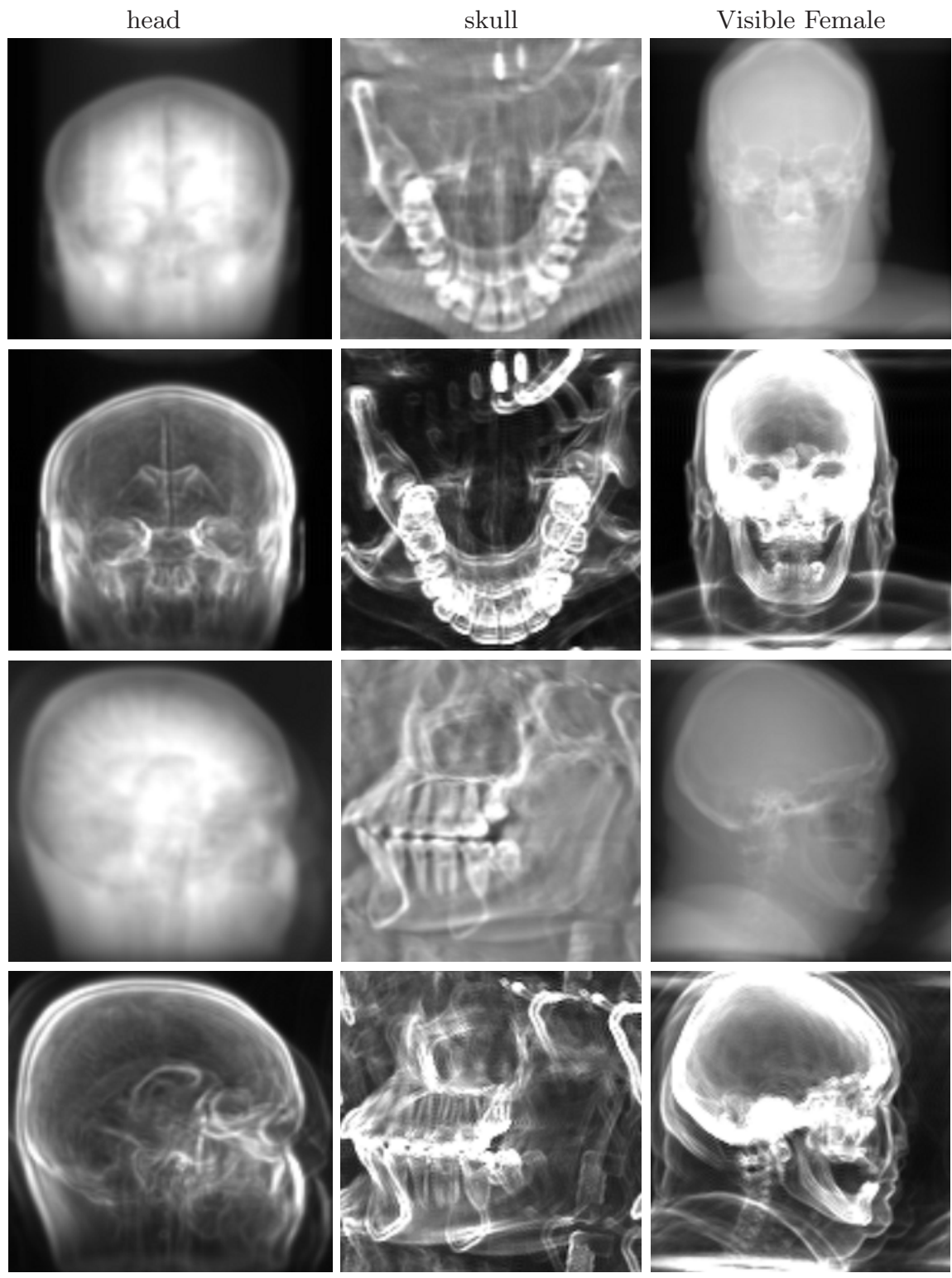

Fig. 1. Rendering results. We exemplify our method on three datasets, namely the head ( $1^{\text {st }}$ col. $)$, skull $\left(2^{\text {nd }}\right.$ col.) and Visible Female dataset $\left(3^{r d}\right.$ col. $) .1^{\text {st }}$ and $3^{\text {rd }}$ row: conventional FVR. $2^{\text {nd }}$ and $4^{\text {th }}$ row: respective counterparts of our method using contour extraction. 
From a qualitative point of view, we introduced three types of applications in medical visualization: recovery of hidden features, noise removal, and detection of boundaries. This type of exploration is especially valuable, when the radiologist works with X-ray like projections of the input material. We also have to mention, that the results can not be obtained by applying image processing operators on the respective projections, since high material density mostly leads to a cumulation and hiding of underlying $3 \mathrm{D}$ features, resulting in projections with more feature-less, homogeneous areas.

From a theoretical point of view, we created a Fourier projection algorithm, which is capable of lighting features in the dataset in dependence of the local sample values at interactive rates without the requirement to recalculate the dataset for every view. This is a remarkable property of our algorithm, since spatial properties are normally difficult to localize in Fourier space. We also proved that the first three non-zero coefficients of the SH expansion are sufficient to represent the contour extraction function with an accuracy of about $94.7 \%$.

\section{References}

1. Csébfalvi, B., Gröller, E.: Interactive volume rendering based on a "bubble model". Graphics Interface. (2001)

2. Csébfalvi, B., Mroz, L., Hauser, H., König, A., Gröller, E.: Fast visualization of object contours by non-photorealistic volume rendering. EUROGRAPHICS. (2001).

3. Entezari, A., Scoggins, R., Möller, T., Machiraju, R.: Shading for Fourier Volume Rendering. IEEE Volume Visualization. (2002)

4. Guthe, S., Wand, M., Gonser, J., Strasser, W.: Interactive Rendering of Large Volume Data Sets. IEEE Vsiualization. (2002)

5. Gröbner, W., Hofreiter, N.: Integraltafel, Zweiter Teil, Bestimmte Integrale. 5. Auflage. Springer Verlag. (1973)

6. Kajiya, J.T.: Ray Tracing Volume Densities. ACM SIGGRAPH. (1984) 165-174

7. Kniss, J. Premože, S. Hansen, C., Ebert, D. Interactive Translucent Volume Rendering and Procedural Modeling. IEEE Visualization. (2002)

8. Levoy, M.: Volume Rendering using the Fourier projection-slice theorem. Graphics Interface. (1992) 61-69

9. Malzbender, T.: Fourier Volume Rendering. ACM Transactions on Graphics 12 (3), July 1993. (1993) 233-250

10. Ramamoorthi, R., Hanrahan, P.: An Efficient Representation for Irradiance Environment Maps. ACM SIGGRAPH. (2001) 497-500

11. Ramamoorthi, R., Hanrahan, P.: On the Relationship Between Radiance and Irradiance: Determining the Illumination from Images of a Convex Lambertian Object. J. Opt. Soc, Vol.18, No.10, October 2001.

12. Roettger, S., Guthe, S., Weiskopf, D., Ertl, T., Strasser, W.: Smart HardwareAccelerated Volume Rendering. Joint EUROGRAPHICS-IEEE TVCG Symposium on Visualization. (2003)

13. Totsuka, T., Levoy, M.: Frequency domain volume rendering. Computer Graphics, 27:(4), August 1993. 271-278

14. Varshalovich, D.A., Moskalev, A.N., Khersonskii, V.K.: Quantum Theory of Angualar Momentum. Irreducible Tensors, Spherical Harmonics, Vector Coupling Coefficients, 3nj Symbols. World Scientific. (1988) 130-163 


\section{A Appendix}

\section{A.1 Explicit Formula for $f_{l 0}$}

$f_{l 0}$ can be obtained using equation (5) on p. 143 of 14 by specialization setting $f(\theta, \phi)=f(\theta)=(\sin \theta)^{2 k}$, and by observing that $Y_{l 0}^{*}(\theta)=Y_{l 0}(\theta)=P_{l}(\cos \theta)$ :

$$
f_{l 0}=\sqrt{\frac{2 l+1}{4 \pi}} \int_{0}^{2 \pi} \int_{0}^{\pi}(\sin \theta) f(\theta) Y_{l 0}(\theta) d \phi d \theta=\sqrt{\pi(2 l+1)} \int_{0}^{\pi}(\sin \theta)^{2 k+1} P_{l}(\cos \theta) d \theta,
$$

where $P_{l}(x)$ is the Legendre polynomial of the first kind. By plugging $x=\cos \theta$ into Equation (7) on p. 24 of [5] we obtain

$$
\int_{-1}^{1} x^{2 k} P_{l}(x) d x=\int_{0}^{\pi} \sin \theta(\cos \theta)^{2 k} P_{l}(\cos \theta) d \theta=\left\{\begin{array}{cc}
0 & 2 k<l \text { or } l \text { odd } \\
\frac{2(2 k-l+2 ; 1 ; l)}{(2 k-l+3 ; 2 ; l)} & \text { otherwise }
\end{array}\right.
$$

where $(m ; d ; \nu):=m(m+d)(m+2 d) \ldots(m+(\nu-1) d)$. Thus, using the relationship $\sin \theta=\sqrt{1-\cos ^{2} \theta}$ and the binomial theorem, we obtain for $2 k \geq l$ and $l$ even

$$
\begin{aligned}
f_{l 0} & =\sqrt{\pi(2 l+1)} \int_{0}^{\pi}(\sin \theta)^{2 k+1} P_{l}(\cos \theta) d \theta=\sqrt{\pi(2 l+1)} \int_{0}^{\pi} \sin \theta\left(1-\cos ^{2} \theta\right)^{k} P_{l}(\cos \theta) d \theta \\
& =\sqrt{\pi(2 l+1)} \sum_{n=0}^{k}(-1)^{n}\left(\begin{array}{l}
k \\
n
\end{array}\right) \int_{0}^{\pi} \sin \theta\left(\cos ^{2 n} \theta\right) P_{l}(\cos \theta) d \theta \\
& =\sqrt{\pi(2 l+1)} \sum_{n=0}^{k}(-1)^{n}\left(\begin{array}{l}
k \\
n
\end{array}\right) \frac{2(2 n-l+2 ; 1 ; l)}{(2 n-l+3 ; 2 ; l)}
\end{aligned}
$$

and zero, otherwise.

\section{A.2 Quality of the Approximation of $f(\theta)$}

The Parseval condition (see p. 144 in [14) states, that

$$
F:=\sum_{l=0}^{\infty} \sum_{m=-l}^{l}\left|f_{l m}\right|^{2}=\int_{0}^{2 \pi} \int_{0}^{\pi}|f(\theta, \phi)|^{2} \sin \theta d \phi d \theta
$$

If $\mathrm{F}$ is finite, the residual error can be determined from this formula when $f(\theta, \phi)$ is approximated with the first t terms only. Plugging the analytical expression for $\mathrm{f}$ into equation (13) leads to the residual error

$$
R:=\int_{0}^{2 \pi} \int_{0}^{\pi}(\sin \theta)^{4 k+1} d \phi d \theta-\sum_{l=0}^{t}\left|f_{l 0}\right|^{2}
$$

In particular, for $\mathrm{k}=8$ and $\mathrm{t}=4$, we obtain $\mathrm{F}=2.720966695$ and $\mathrm{R}=2.576686223$, thus $94.7 \%$ of the energy is contained in the expansion using the first three non-zero coefficients. 